\title{
Em Memória: Professor José Silvério Santos Diniz (1935-2011)
}

In Memoriam: Professor José Silvério Santos Diniz (1935-2011)

\section{Autores}

Eduardo Araújo

Oliveira $^{1}$

Ana Cristina Simões e

Silva ${ }^{1}$

Eleonora Moreira Lima ${ }^{1}$

José Maria Penido

Silva ${ }^{1}$

'Departamento de

Pediatria, Faculdade

de Medicina da

Universidade Federal de

Minas Gerais - UFMG.

Data de submissão: 15/06/2010 Data de aprovação: 28/07/2011

Correspondência para: Eduardo Araújo Oliveira Rua Engenheiro Amaro Lanari, 389/501

Belo Horizonte (MG) - Brasil CEP: 30310-580

E-mail: eduolive@ medicina.ufmg.br

O referido estudo foi realizado na UFMG.

Os autores declaram a inexistência de conflitos de interesse.

\section{Resumo}

Em 23 de maio de 2011, faleceu o Professor José Silvério Santos Diniz, um dos expoentes da Nefrologia Pediátrica brasileira. Este editorial descreve de forma sumária a trajetória desse grande professor, médico e pesquisador de nosso País.

Palavras-chave: Pediatria. Ensino. Pesquisa. Nefrologia.

\section{INTRODUÇÃO}

"If I have seen further it is by standing on the shoulders of giants". (Newton, 1676).

Em 23 de maio de 2011, faleceu o Professor José Silvério Santos Diniz, um dos expoentes da Nefrologia Pediátrica brasileira.

José Silvério Santos Diniz, conhecido por todos como Silvério, nasceu em 03 de dezembro de 1935, em Miguel Burnier, município de Ouro Preto, onde viveu até três meses de idade, quando seus pais se mudaram para Conselheiro Lafayette. Passou a infância lá e adolescente voltou para Ouro Preto, onde estudou no Colégio Arquidiocesano. Após isso, mudou-se para Belo Horizonte, estudando no antigo Colégio Mineiro, hoje chamado de Colégio Estadual. Graduou-se em Medicina pela Faculdade de Ciências Médicas em 1960. Durante o curso de Medicina, para sua própria manutenção, trabalhou inicialmente como escriturário na prefeitura de Belo Horizonte. Em 1957, também por concurso, passou para o cargo de laboratorista de análises clínicas da prefeitura de Belo Horizonte.

\section{Abstract}

Professor José Silvério Santos Diniz, an exponent of the Brazilian Pediatric Nephrology, died on 23 May, 2011. This editorial is a brief description of the career of this great Brazilian teacher, physician and researcher.

Keywords: Pediatrics. Teaching. Research. Nephrology.

Casou-se com a professora Thais Flores Nogueira Diniz em 1961. Tiveram quatro filhos: Leonardo (engenheiro), Guilherme (médico pediatra), Susana (bióloga e professora universitária) e Virgílio (advogado).

De 1961 a 1964, trabalhou no interior de Minas Gerais, em Acesita, como médico generalista. Essa atividade teve um caráter formativo. As experiências vividas nesse período foram de suma importância para as reflexões de toda sua vida científica e humana.

Em 1964, foi selecionado pela então Cátedra de Pediatria da Faculdade de Medicina (FM) da Universidade Federal de Minas Gerais (UFMG) para exercer as funções de instrutor de ensino. Em 1969, tornou-se professor assistente. Concluiu a livre docência em 1977, na UFMG, com o trabalho 'Síndrome Nefrótica na Criança. Estudo evolutivo da Nefrose Lipoídica e sua correlação anátomo-clínica'. Posteriormente, por concurso, foi a professor adjunto em 1978 e, em seguida, em 1982, também por concurso, a professor titular. Aposentou-se em 1994 e recebeu o título de Professor Emérito da FM da UFMG em 1997 (Figura 1). 
Figura 1. Professor José Silvério Santos Diniz. Quadro de Professor Emérito da Universidade Federal de Minas Gerais (1997). Acervo do Centro de Memória da Faculdade de Medicina da UFMG.

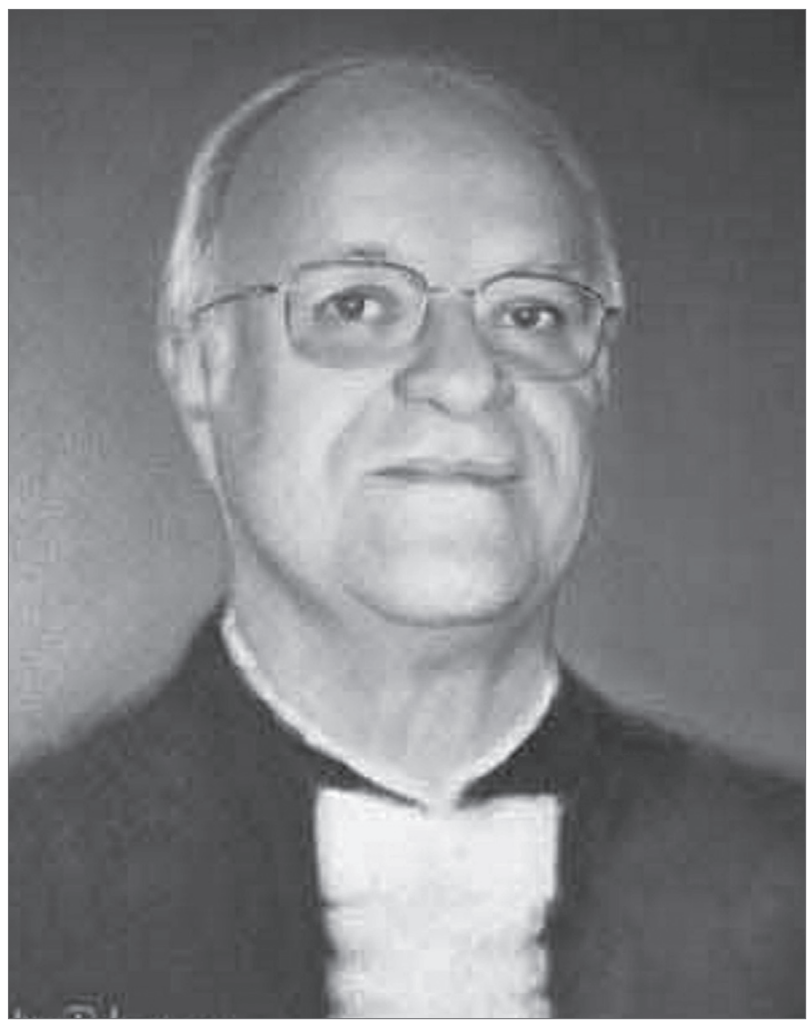

\section{Contribuição InSTITUCIONAL}

Durante sua carreira acadêmica, dedicou-se, com entusiasmo, às atividades de ensino (graduação e pós-graduação), pesquisa e assistência, as bases formadoras de um médico. Para melhor exercer as atividades administrativas, fez uma pós-graduação em Administração Hospitalar, formando-se em 1967. Exerceu diversos cargos administrativos de relevância na Faculdade de Medicina na UFMG e no Hospital das Clínicas (HC) da UFMG.

A partir de 1968, interessado em melhor cuidar das crianças internadas na enfermaria de pediatria do HC, a grande maioria com diagnóstico de infecção urinária, passou a se interessar pelo estudo das doenças do trato urinário. Nessa ocasião, teve a ideia de formar um grupo de nefrologia pediátrica. Assim, desde então, dedicouse à pesquisa na área de Nefrologia Pediátrica, tendo fundado a Unidade de Nefrologia Pediátrica, vinculada ao Departamento de Pediatria e também conhecida como Grupo de Rim (GRIM), que cresceu e se solidificou através dos anos, hoje composta por oito doutores, um especialista em Nefrologia Pediátrica e vários mestrandos e doutorandos. Em 1972, o Prof. Silvério fez um estágio no Serviço de Nefrologia Pediátrica da
Figura 2. Confraternização de final de ano da Unidade de Nefrologia Pediátrica (dezembro de 2010). Da esquerda para a direita, na primeira fila: Professora Mônica Maria de Vasconcelos, Professora Ana Cristina Simões e Silva, Professora Eleonora Moreira Lima, Professor Eduardo Araújo Oliveira e Professor José Silvério Santos Diniz. Da esquerda para a direita, na fila posterior: Professor Luiz Sérgio Bahia Cardoso, Professor José Maria Penido Silva e Professor Sérgio Veloso Brant Pinheiro.

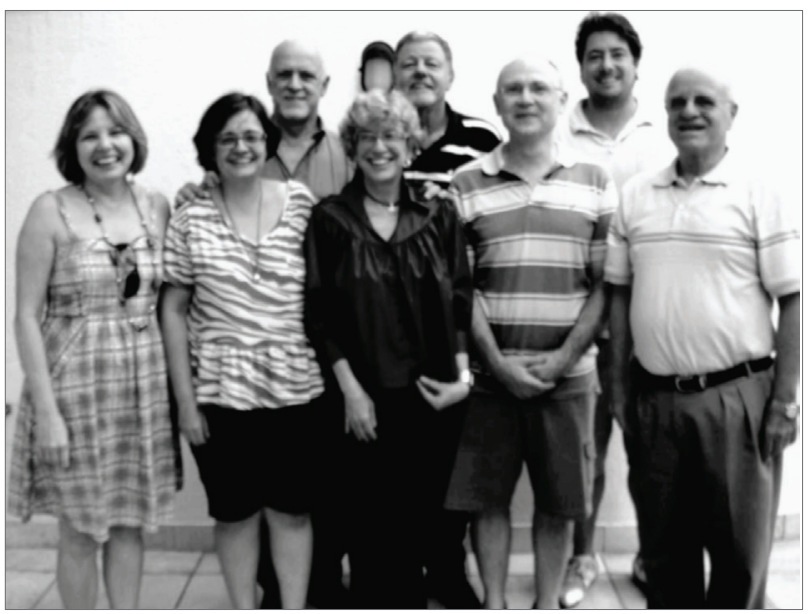

Santa Casa de Misericórdia de São Paulo com o Prof. Julio Toporovski, o primeiro nefrologista pediátrico brasileiro. Com a ajuda dos companheiros, iniciou a estrutura de cadastramento dos pacientes compilando ou anotando seus nomes e registros em livro próprio. Dessa iniciativa, nasceu o cadastro dos pacientes do Grupo de Rim, que hoje já está na faixa dos 7.000 pacientes cadastrados com prontuário específico, possibilitando à Unidade de Nefrologia Pediátrica executar vários estudos e ser reconhecida no Brasil e no exterior como um grupo que contribui para o aumento do conhecimento nessa área (Figura 2).

No início, as dificuldades eram muito grandes, pois, além de um desconhecimento teórico por parte de todos, também não se tinha vivência da abordagem clínica. Era obrigatório enfrentar o desafio de não somente atender cada vez melhor as crianças nefrouropatas, mas também ampliar os conhecimentos e divulgá-los. O grupo, sob seu comando, foi sempre receptivo aos alunos interessados e aos médicos ou residentes que quisessem dele participar. Assim, passaram pelo grupo vários membros que, após grande contribuição, encontraram outros caminhos de atuação. Outros nele permaneceram, consolidando a estrutura de ensino pesquisa e extensão.

A Unidade, internacionalmente conhecida, desenvolve atividades na graduação na formação do médico generalista, médico especialista em nefrologia 
pediátrica, pós-graduação (residência, mestrado, doutorado), assistência e pesquisa, compreendendo diversas linhas, com inúmeros trabalhos científicos publicados, que com muita satisfação poderão ser consultados muito em breve, quando lançaremos o site da Unidade que já está em fase de testes. A partir de 1990, Silvério organizou o 'Programa interdisciplinar de prevenção e assistência na insuficiência renal crônica em crianças e adolescentes', constituído por médicos (nefrologistas pediátricos, pediatras e urologistas), enfermeiros, nutricionista, assistente social e psicólogo, com o objetivo de prestar assistência ambulatorial a crianças e adolescentes portadores de doença renal crônica em tratamento conservador. Esse programa antecipou-se aos diversos centros de nefrologia no Brasil, confirmando que o atendimento adequado para as crianças portadoras de doenças renais deve ter um caráter interdisciplinar. Em pleno funcionamento, esse programa representa uma combinação estruturada de assistência e pesquisa, solidificando-se em atividade de ensino, pesquisa e extensão de excelência em área de alta complexidade da Nefrologia Pediátrica. Ainda nesse contexto, paralelamente, o Prof. Silvério estimulou e trabalhou arduamente para a constituição do Centro de Diálise do Hospital das Clínicas, culminando, na década de 90, com a implantação do programa de diálise peritoneal contínua que tantos benefícios têm trazido no tratamento de jovens crianças com doença renal crônica em estádio final.

Nos últimos anos, voltou-se para a pesquisa na área do refluxo vesicoureteral, com ênfase à Biologia Molecular, objetivo de seus estudos no último pósdoutorado em Londres em 2003 e 2004. Uma das características marcantes do Professor Silvério foi sua preocupação com a atualização científica, o intercâmbio com centros de estudos avançados no Brasil e no exterior. Nesse contexto, sempre procurou trazer pesquisadores de renome internacional, como o Professor Martin Barrat do Institute of Child Health (Londres), para uma troca de experiências e informações. Nesse sentido, também realizou seis estágios de pós-doutorado, de média e longa duração, em Londres e em Indianápolis.

Além dessa vigorosa atividade acadêmica, o Prof. Silvério teve uma participação extensa na administração universitária, contribuindo com sua experiência e dinamismo para o desenvolvimento da instituição. Entre os diversos cargos, podemos enumerar: Coordenador do Colegiado de Curso de Graduação em Medicina (1972/1973), Chefe do Departamento de Pediatria da Faculdade de Medicina da UFMG (1980/1984) e Diretor do Hospital das Clínicas das
UFMG (1986/1988). Nesse cargo, ampliou as unidades de internação e deu continuidade à reforma do prédio central. Nesse período também incentivou o surgimento e trabalhou para consolidar diversas clínicas emergentes, como a então incipiente Medicina Fetal, vinculada ao Departamento de Ginecologia e Obstetrícia.

Por sua destacada atuação acadêmica, o professor Silvério recebeu o reconhecimento da sociedade por meio de vários títulos e honrarias, como:

- Foi paraninfo duas vezes de turmas de Graduação em Medicina da FM da UFMG (1982/1983).

- Recebeu a medalha Santos Dumont do Governo do Estado de Minas Gerais (1986).

- Recebeu homenagem da Prefeitura e Câmara Municipal de Conselheiro Lafaiete (MG) por sua destacada atuação fora de sua cidade (1990).

- Foi eleito Membro da Academia Mineira de Medicina - Cadeira de número 41 (1992).

- Foi presidente da Sociedade Mineira de Pediatria (1997).

- Recebeu Certificado de Personalidade Médica do Ano - Associação Médica de Minas Gerais (1998).

- Foi Presidente de Honra do XI Congresso Brasileiro de Nefrologia Pediátrica, Sociedade Brasileira e Mineira de Nefrologia (2001).

- Foi eleito Membro da Academia Mineira de Pediatria (2006)

\section{Contribuição CIEntíFICA}

Além da insuperável contribuição institucional nas mais variadas instâncias da vida acadêmica na UFMG, incluindo um fundamental papel no Departamento de Pediatria, na FM e no HC, durante a sua carreira, o Professor Silvério também contribuiu com seus trabalhos para a compreensão de diversas doenças renais em crianças e adolescentes de nosso meio. Suas contribuições são particularmente importantes e originais em várias áreas. Podemos destacar alguns tópicos pioneiramente abordados pelo Professor José Silvério e que posteriormente se tornaram robustas linhas de pesquisa de nossa Unidade de Nefrologia Pediátrica: (1) contribuição para a compreensão da correlação anátomo-patológica da síndrome nefrótica em crianças; (2) contribuição para o conhecimento da evolução do tratamento conservador do refluxo vesicoureteral e da etiopatogenia da nefropatia do refluxo; (3) no final da década de 80 , foi pioneiro no relato dos primeiro casos de diagnóstico pré-natal das anomalias do trato urinário, criando o Ambulatório de Hidronefrose Fetal, em plena atividade nos dias de hoje com mais de 800 pacientes acompanhados; (4) implantação do 
Programa Interdisciplinar de Prevenção e Tratamento da Doença Renal Crônica em Crianças e Adolescentes no início da década de 90.

Como já mencionado, esse projeto de característica interdisciplinar foi desenvolvido sob sua coordenação e contou inicialmente com o apoio financeiro da Coordenação de Aperfeiçoamento de Pessoal de Nível Superior (CAPES), após ter sido selecionado pela Próreitoria de Pós-graduação da Universidade Federal de Minas Gerais (PRPg-UFMG). Pode-se afirmar que o Programa modificou positivamente a abordagem clínica das crianças e adolescentes com DRC em nossa Unidade e foi pioneiro na UFMG no que se refere à utilização de abordagem multi e interdisciplinar para o cuidado de pacientes pediátricos portadores de doenças crônicas. Hoje, o panorama é completamente distinto daquele da década de 1980, quando as crianças com DRC permaneciam internadas na enfermaria do HC-UFMG por meses e vinham a falecer após intenso sofrimento para eles e suas famílias (e para a equipe médica).

Essa intensa e inquieta busca do conhecimento pelo Professor Silvério desdobrou-se em uma produção científica vigorosa e publicações em periódicos de alto impacto e de grande prestígio na comunidade científica pediátrica, assim como da nefrologia e urologia, como Journal of Urology, The Journal of Pediatrics, Pediatric Nephrology, Pediatric Research, BJU International, Pediatric Infectious Disease Journal, Jornal de Pediatria e Jornal Brasileiro de Nefrologia, entre outros. ${ }^{1-36}$ No total, foram 75 artigos completos publicados em periódicos nacionais e internacionais, 30 capítulos de livros e aproximadamente 300 apresentações em congressos (http:// lattes.cnpq.br/6272691744956776). Em relação ao impacto dos artigos, na base de dados Web of Science (http://science.thomsonreuters.com), foram encontrados 25 artigos indexados com 219 citações, média de 8,7 citações por artigo, com índice $\mathrm{H}$ de 10 , e na base Scopus (http://www.scopus.com), foram encontrados 36 documentos, com um total de 319 citações e média de 8,8 citações por artigo, atingindo índice $\mathrm{H}$ de 12. É importante ressaltar que as publicações de maior impacto do Prof. Silvério incluem ampla gama de assuntos pertinentes à Nefrologia Pediátrica, como, por exemplo, refluxo vesicoureteral, anomalias congênitas do trato urinário, hidronefrose fetal, síndrome nefrótica, infecção urinária, hipertensão, entre outros.

Além dessa notável produção científica, o Professor Silvério se destacou na formação de recursos humanos de qualidade para a prática pediátrica e da nefrologia pediátrica. Com seu dinamismo e sua personalidade agregadora, procurava atrair jovens com as mais diversas características e ecléticas capacidades, vislumbrando compor um grupo unido, humano e ético do ponto de vista assistencial e, ao mesmo tempo, capaz de produzir conhecimento de qualidade. Nesse contexto, além das inúmeras orientações informais não contabilizadas, mais de 30 bolsistas de iniciação científica (bolsas do CNPq e da FAPEMIG), o Professor Silvério orientou no programa de Pósgraduação Ciências da Saúde - Saúde da Criança e do Adolescente da FM da UFMG cinco dissertações de mestrado e três teses de doutorado. Ressalta-se nesse grupo de orientandos que todos são atualmente (ou foram) professores do Departamento de Pediatria da FM da UFMG e muitos mantêm a tradição, sendo orientadores de outros jovens candidatos à prática da nefrologia pediátrica e da pediatria (Quadro 1).

\section{Quadro 1 Orientações de Pós-graduação (mestrado e doutorado) realizadas pelo Professor José} Silvério Santos Diniz e destino atUal dos alunos ORIENTADOS PELO REFERIDO PROFESSOR.

\begin{tabular}{|c|c|c|}
\hline Aluno & Nível - ano & Destino atual \\
\hline José Maria Penido Silva & Mestrado 1987 & $\begin{array}{l}\text { Professor Adjunto do Depto. Pediatria - UFMG } \\
\text { Coordenador da Unidade Nefrologia Pediátrica 1994-2009 }\end{array}$ \\
\hline \multirow{2}{*}{ Eduardo Araujo Oliveira } & Mestrado 1992 & Professor Titular do Depto. Pediatria - UFMG \\
\hline & Doutorado 1998 & Coordenador da Unidade Nefrologia Pediátrica \\
\hline Viviane Santuari P. Marino & Mestrado 1993 & Professora Adjunta do Depto. Patologia Clínica - UFMG \\
\hline Maria Goretti Moreira Guimarães Penido & Mestrado 1995 & $\begin{array}{l}\text { Professora Adjunta do Depto. Pediatria - UFMG } \\
\text { Membro da Unidade Nefrologia Pediátrica }\end{array}$ \\
\hline Mônica Maria de Almeida Vasconcelos & Mestrado 1999 & $\begin{array}{l}\text { Professora Adjunta Depto. Pediatria - UFMG } \\
\text { Membro da Unidade Nefrologia Pediátrica }\end{array}$ \\
\hline Ana Cristina Simões e Silva & Doutorado 2000 & $\begin{array}{l}\text { Professora Titular do Depto. Pediatria - UFMG } \\
\text { Coordenadora do Programa de Pós-graduação em Saúde } \\
\text { da Criança e do Adolescente }\end{array}$ \\
\hline Roberto de Assis Ferreira & Doutorado 2000 & Professor Emérito do Depto. Pediatria - UFMG \\
\hline
\end{tabular}


Em linhas gerais, essas são as principais contribuições científicas do Professor José Silvério Santos Diniz que podem ser enumeradas do ponto de vista quantitativo. Contudo, é inegável que suas contribuições para a Pediatria e para a Nefrologia Pediátrica vão muito além do que a natureza fria dos números permite julgar. Como um professor engajado nas mais importantes questões do ensino, da pesquisa, da assistência médica e da saúde pública, o Professor Silvério nunca se furtou ao debate de ideias e dos avanços nas mais diversas dimensões da vida universitária. É inesquecível para quem teve a feliz oportunidade da convivência com o Professor Silvério a participação nos debates acalorados e sua apaixonada defesa dos pontos de vista que julgava mais apropriados, sempre em defesa dos pacientes e dos seus familiares. São marcantes as características que permeavam essas discussões, nas quais ele sempre se pautava por uma visão humanística e ética da prática médica e da prática da ciência.

Acreditamos que a comunidade da Nefrologia Pediátrica brasileira está de luto pela irreparável lacuna deixada pela morte do Professor José Silvério Santos Diniz. Compartilhamos a dor com sua amada esposa Thaís, seus filhos Leonardo, Guilherme, Virgílio e Suzana e seus seis netos. Fica a certeza de um legado inestimável pelo exemplo, pelo dinamismo, pela alegria de viver compartilhada pelos familiares, amigos e colaboradores. Fica uma missão para a Unidade de Nefrologia Pediátrica (HC-UFMG): honrar esse legado, pautando a sua atuação pelos altos parâmetros fixados pelo Professor Silvério.

\section{REFERÊNCIAS}

1. Diniz JS. Aspects of Brazilian paediatric nephrology. Pediatr Nephrol 1988;2:271-6.

2. Diniz JS, Silva JM, Silva AF, Junior MM, Heringer SB, Batista EN, et al. [Hypertension: retrospective study in children from a pediatric nephrology unit]. J Pediatr (Rio J) 1994;70:226-33.

3. Oliveira EA, Cabral AC, Leite HV, Rabêlo EA, Colosimo EA, Oliveira RB, et al. [Fetal hydronephrosis: postnatal management and follow up]. J Pediatr (Rio J) 1997;73:252-8

4. Oliveira EA, Diniz JS, Silva JM, Rabelo EA, Pontes AK, Souza MF. Features of primary vesicoureteric reflux detected by investigation of foetal hydronephrosis. Int Urol Nephrol 1998;30:535-41.

5. Oliveira EA, Diniz JS, Cabral AC, Leite HV, Colosimo $\mathrm{EA}$, Oliveira RB, et al. Prognostic factors in fetal hydronephrosis: a multivariate analysis. Pediatr Nephrol 1999;13:859-64.

6. Diniz JS, Oliveira EA, Servilha MM. [Diabetes insipidus as an early clinical manifestation of pineal tumor]. J Pediatr (Rio J) 2000;76:383-6.
7. Oliveira EA, Diniz JS, Cabral AC, Pereira AK, Leite HV, Colosimo EA, et al. Predictive factors of fetal urethral obstruction: a multivariate analysis. Fetal Diagn Ther 2000;15:180-6

8. Oliveira EA, Diniz JS, Rabelo EA, Silva JM, Pereira AK, Filgueiras MT, et al. Primary megaureter detected by prenatal ultrasonography: conservative management and prolonged follow-up. Int Urol Nephrol 2000;32:13-8.

9. Oliveira EA, Cabral AC, Pereira AK, Machado IN, Diniz JS, Lana AM, et al. Outcome of fetal urinary tract anomalies associated with multiple malformations and chromosomal abnormalities. Prenat Diagn 2001;21:129-34.

10. Oliveira EA, Diniz JS, Vilasboas AS, Rabelo EA, Silva JM, Filgueiras MT. Multicystic dysplastic kidney detected by fetal sonography: conservative management and follow-up. Pediatr Surg Int 2001;17:54-7.

11. Penido MG, Diniz JS, Moreira ML, Tupinambá AL, França A, Andrade BH, et al. [Idiopathic hypercalciuria: presentation of 471 cases]. J Pediatr (Rio J) 2001;77:101-4.

12. Oliveira EA, Rabelo EA, Pereira AK, Diniz JS, Cabral AC, Leite HV, et al. Prognostic factors in prenatallydetected posterior urethral valves: a multivariate analysis. Pediatr Surg Int 2002;18:662-7.

13. Penido MG, Diniz JS, Guimarães MM, Cardoso RB, Souto MF. [Urinary excretion of calcium, uric acid and citrate in healthy children and adolescents]. J Pediatr (Rio J) 2002;78:153-60.

14. Simões e Silva AC, Oliveira EA, Gomes CR, Lima FS, Diniz JS. [Liddle's syndrome: late diagnosis of a rare cause of arterial hypertension]. J Pediatr (Rio J) 2002;78:251-4.

15. Apocalypse GT, Oliveira EA, Rabelo EA, Diniz JS, Marino VS, Pereira AK, et al. Outcome of apparent ureteropelvic junction obstruction identified by investigation of fetal hydronephrosis. Int Urol Nephrol 2003;35:441-8.

16. Silva JM, Diniz JS, Oliveira EA, Cardoso LS, Marino VS, Pimenta MR, et al. Features of primary vesicoureteral reflux and renal damage in children at a single institution in Brazil from 1969 to 1999. Int Urol Nephrol 2003;35:161-8.

17. Soares CM, Oliveira EA, Diniz JS, Lima EM, Vasconcelos MM, Oliveira GR. Predictive factors of progression of chronic renal insufficiency: a multivariate analysis. Pediatr Nephrol 2003;18:371-7.

18. Rabelo EA, Oliveira EA, Diniz JS, Silva JM, Filgueiras MT, Pezzuti IL, et al. Natural history of multicystic kidney conservatively managed: a prospective study. Pediatr Nephrol 2004;19:1102-7.

19. Silva JM, Diniz JS, Oliveira EA, Marino VS, Pimenta MR, Matos CC, et al. [Baseline clinical features of 483 children and adolescents with primary vesicoureteral reflux: a retrospective study]. Rev Assoc Med Bras 2004;50:153-7.

20. Simões e Silva AC, Diniz JS, Regueira Filho A, Santos RA. The renin angiotensin system in childhood hypertension: selective increase of angiotensin-(1-7) in essential hypertension. J Pediatr 2004;145:93-8. 
21. Diniz JSS, Silva JMP, Lima EM, Cardoso LS, Moreira ML, Silva AC, et al. 30 anos de Experiência em Nefrologia Pediátrica: um Estudo Descritivo. J Bras Nefrol 2005;27:201-6.

22. Abrantes MM, Cardoso LS, Lima EM, Penido Silva JM, Diniz JS, Bambirra EA, et al. Predictive factors of chronic kidney disease in primary focal segmental glomerulosclerosis. Pediatr Nephrol 2006;21:1003-12.

23. Abrantes MM, Cardoso LS, Lima EM, Silva JM, Diniz JS, Bambirra EA, et al. Clinical course of 110 children and adolescents with primary focal segmental glomerulosclerosis. Pediatr Nephrol 2006;21:482-9.

24. Penido Silva JM, Oliveira EA, Diniz JS, Bouzada MC, Vergara RM, Souza BC. Clinical course of prenatally detected primary vesicoureteral reflux. Pediatr Nephrol 2006;21:86-91.

25. Silva JM, Diniz JS, Lima EM, Vergara RM, Oliveira EA. Predictive factors of resolution of primary vesico-ureteric reflux: a multivariate analysis. BJU Int 2006;97:1063-8.

26. Silva JM, Diniz JS, Silva AC, Azevedo MV, Pimenta MR, Oliveira EA. Predictive factors of chronic kidney disease in severe vesicoureteral reflux. Pediatr Nephrol 2006;21:1285-92.

27. Silva JM, Oliveira EA, Diniz JS, Cardoso LS, Vergara RM, Vasconcelos MA, et al. Gender and vesicoureteral reflux: a multivariate analysis. Pediatr Nephrol 2006;21:510-6.

28. Simões e Silva AC, Diniz JS, Pereira RM, Pinheiro SV, Santos RA. Circulating renin Angiotensin system in childhood chronic renal failure: marked increase of Angiotensin-(1-7) in end-stage renal disease. Pediatr Res 2006;60:734-9.

29. Simões e Silva AC, Silva JM, Diniz JS, Pinheiro SV, Lima EM, Vasconcelos MA, et al. Risk of hypertension in primary vesicoureteral reflux. Pediatr Nephrol 2007;22:459-62.

30. Soares CM, Diniz JS, Lima EM, Silva JM, Oliveira GR, Canhestro MR, et al. Clinical outcome of children with chronic kidney disease in a pre-dialysis interdisciplinary program. Pediatr Nephrol 2008;23:2039-46.

31. Silva JM, Diniz JS, Lima EM, Pinheiro SV, Marino VP, Cardoso LS, et al. Independent risk factors for renal damage in a series of primary vesicoureteral reflux: a multivariate analysis. Nephrology (Carlton) 2009;14:198-204.

32. Soares CM, Diniz JS, Lima EM, Oliveira GR, Canhestro MR, Colosimo EA, et al. Predictive factors of progression to chronic kidney disease stage 5 in a predialysis interdisciplinary programme. Nephrol Dial Transplant 2009;24:848-55.

33. Dias CS, Silva JM, Diniz JS, Lima EM, Marciano RC, Lana LG, et al. Risk factors for recurrent urinary tract infections in a cohort of patients with primary vesicoureteral reflux. Pediatr Infect Dis J 2010;29:139-44.

34. Marciano RC, Soares CM, Diniz JS, Lima EM, Silva JM, Canhestro MR, et al. Mental disorders and quality of life in pediatric patients with chronic kidney disease. J Bras Nefrol 2010;32:316-22.

35. Marciano RC, Soares CM, Diniz JS, Lima EM, Silva JM, Canhestro MR, et al. Behavioral disorders and low quality of life in children and adolescents with chronic kidney disease. Pediatr Nephrol 2011;26:281-90.

36. Quirino IG, Silva JM, Diniz JS, Lima EM, Rocha AC, Simões e Silva AC, et al. Combined use of late phase dimercapto-succinic acid renal scintigraphy and ultrasound as first line screening after urinary tract infection in children. J Urol 2011;185:258-63. 